\title{
Neutrophil-to-Lymphocyte Ratio and Intratumoral CD45RO- Positive T Cells as Predictive Factors for Longer Survival of Patients with Colorectal Liver Metastasis after Hepatectomy
}

\author{
Takeomi Hamada, ${ }^{1}$ Hidenobu Ishizaki, ${ }^{1}$ Yukihiro Haruyama, ${ }^{2}$ Roko Hamada, ${ }^{1}$ \\ Koichi Yano, ${ }^{1}$ Kazuhiro Kondo, ${ }^{1}$ Hiroaki Kataoka ${ }^{2}$ and Atsushi Nanashima ${ }^{1}$ \\ ${ }^{1}$ Department of Surgery, University of Miyazaki Faculty of Medicine, Miyazaki, Miyazaki, Japan \\ ${ }^{2}$ Department of Pathology, University of Miyazaki Faculty of Medicine, Miyazaki, Miyazaki, Japan
}

\begin{abstract}
Colorectal cancer is the fourth most common malignancy across the world, and over $50 \%$ of patients had colorectal liver metastases (CLM). Activated neutrophils and tumor-infiltrating lymphocytes (TILs) are considered to interrupt progression of primary colorectal cancer; however, immunological host reactions to CLM have not been fully elucidated. We thus aimed to explore the prognostic implication of neutrophil-tolymphocyte ratio (NLR) in peripheral blood and TILs in resected metastatic cancer tissues of 29 patients with CLM who underwent hepatectomy. To evaluate local immunological responses in CLM, we examined the infiltration of CD66b+ neutrophils and TILs, such as CD8+ T cells, CD45RO+ T cells, and forkhead box P3+ (FOXP3+) T cells. The presence of fewer than 4 tumors $(p=0.0005)$, the absence of distant metastasis $(p=0.018)$, adjuvant anti-cancer chemotherapy $(p=0.0013)$, and elevated NLR over $4.1(p=$ 0.026) were found to be significant parameters related to longer survival after hepatectomy. Further, high numbers of infiltrated CD45RO+ T cells in CLM were significantly associated with longer patient survival ( $p$ $=0.020)$. The numbers of CD45RO+ T cells were correlated with those of CD8+T cells $(p=0.008)$. The numbers of peripheral blood neutrophils were negatively correlated with those of CD45RO+T cells $(p=$ $0.038)$ and of CD66b+ neutrophils $(p=0.008)$ in CLM. The present data indicate that elevated peripheral blood NLR and high numbers of intratumoral CD45RO+ T cells are predictive of longer CLM patient survival after hepatectomy among current biomarkers.
\end{abstract}

Keywords: colorectal liver metastasis; hepatectomy; host immunological response; survival; tumor-infiltrating lymphocytes

Tohoku J. Exp. Med., 2020 August, 251 (4), 303-311.

\section{Introduction}

Colorectal cancer is the fourth most common malignancy across the world, with approximately 1 million new cases and 500,000 deaths occurring each year (Jemal et al. 2010). A current study estimated that over $50 \%$ of patients with colorectal carcinoma had colorectal liver metastases (CLM). At present, hepatic resection is the only curative treatment for patients with CLM and which provides longterm survival (Beppu et al. 2012).

Various prognostic factors characterizing the malignant biological behaviors of CLM have been reported such as classical clinicopathological tumor factors (size, number, irregularity, location, histological differentiation, vascular infiltration, and primary node metastasis), genetic or chromosomal aberrations, proliferative activity, adhesion molecules, microvessel density, angiogenic factors, tumor released cytokines and others (Popat et al. 2005; Tomlinson et al. 2007; Salazar et al. 2011; Kimura et al. 2016). Immunological host responses against cancer progression or invasion were also recently noted to be well associated with patient survival (Bindea et al. 2010). Systemic or local host immune responses by immune cells, as evidenced by the accumulation of leukocytes, lymphocytes, or fibroblasts surrounding cancer tissue, may inhibit or modulate cancer progression (Galdiero et al. 2013). In primary colorectal carcinomas, the accumulation of immune cells surrounding tumor infiltrations has been shown to play

Received February 19, 2020; revised and accepted July 16, 2020. Published online August 7, 2020; doi: 10.1620/tjem.251.303.

Correspondence: Atsushi Nanashima, M.D., Department of Surgery, University of Miyazaki Faculty of Medicine, 5200 Kihara,

Kiyotake-cho, Miyazaki, Miyazaki 889-1692, Japan.

e-mail: a_nanashima@med.miyazaki-u.ac.jp

(C)2020 Tohoku University Medical Press. This is an open-access article distributed under the terms of the Creative Commons Attribution-NonCommercial-NoDerivatives 4.0 International License (CC-BY-NC-ND 4.0). Anyone may download, reuse, copy, reprint, or distribute the article without modifications or adaptations for non-profit purposes if they cite the original authors and source properly.

https://creativecommons.org/licenses/by-nc-nd/4.0/ 
important roles in suppressing cancer infiltration (Klintrup et al. 2005; Malik et al. 2007; Roxburgh et al. 2009), but the roles of immune cell activity around CLM tissues has not been fully elucidated, to our knowledge. Formation of pseudo-capsular fibrosis around CLM significantly increased survival periods after hepatectomy in a previous report (Lunevicius et al. 2001), and this response is thought to be one of the host immune reactions. The modified Glasgow Prognostic Score (GPS) consists of inflammatory parameters, and the neutrophil-to-lymphocyte ratio (NLR) is a known indicator of systemic immune response that well reflects cancer-associated patient prognosis (McMillan et al. 2007; Halazun et al. 2008; Chang et al. 2014). Meanwhile, in local immune reactions, a subset analysis of accumulated immune cells recently indicated biomarkers that reflect immunological reactions against cancers (Galon et al. 2006; Galdiero et al. 2013; Richards et al. 2014; Stoll et al. 2015). Thus, several prognostic factors predictive of disease-free survival or overall survival (OS) in patients with CLM have been described, and host immune response is an important determinant of outcome in colorectal cancers (Kimura et al. 2016). Biologically malignant behaviors of colon cancers are closely associated with the immune response in the cancer microenvironment, and patient outcome is predominantly governed by the immune response to the primary cancer (Pagès et al. 2005; Galon et al. 2006; Richards et al. 2014). Despite the potential benefit of such evaluations to improve risk stratification for patients with colorectal cancer, a reliable measure of the local inflammatory response has yet to be incorporated into clinical practice (McMillan et al. 2007; Halazun et al. 2008).

We have hypothesized that immune-response would be associated with tumor suppression and the longer survival after curative hepatectomy. Therefore, the present study aimed to examine immunological interactions and clarify survival benefits in CLM patients. We investigated the relationship between patient survival and systemic immune biomarkers of NLR and local immune markers of tumorinfiltrating lymphocytes (TILs) in a series of 29 patients with CLM who underwent hepatectomy at a single cancer institution.

\section{Materials and Methods}

\section{Patients}

Between January 2000 and December 2008, 74 patients with CLM underwent hepatic resection without residual tumors (defined as a curative hepatectomy) at the Department of Surgery, University of Miyazaki Faculty of Medicine (former Department of Surgical Oncology and Regulation of Organ Functions). To exclude an immunological bias for survival, patients who underwent preoperative chemotherapy were excluded in the present series. Resected specimens of the 29 patients were collected for immunohistochemistry analysis. Clinicopathological data were retrospectively examined in these patients. Prognostic variables included 1) factors of the primary lesion such as tumor depth, node metastasis, and dominant histological differentiation; 2) factors of liver metastases comprising the number and size of the tumors and the location and performance of postoperative adjuvant chemotherapy; and 3) factors of host inflammatory responses such as biomarkers measured by routine pre-operative blood tests like hemoglobin, white blood cell counts, serum albumin level, and C-reactive protein. As cancer-specific markers, carcinoembryonic antigen (CEA) and carbohydrate antigen 19-9 (CA19-9) were also examined. Systemic inflammatory response by the modified GPS (mGPS) (McMillan et al. 2007) and systemic immune response as indicated by the NLR (Halazun et al. 2008; Chang et al. 2014) were assessed as preoperative host prognostic factors. Informed consent for this retrospective analysis was obtained from each patient pre-operatively. This study was approved by the institutional review board at the University of Miyazaki in 2013 (Approval number \#2014-028).

\section{Histopathology and immunohistochemical staining}

To evaluate local immune responses in the resected metastatic lesions, immunohistochemical analysis was performed as previously described by A. Ohno, a former staff member in co-author H. Kataoka's laboratory at our institution (Ohno et al., 2014). The selection of specific T-cell subtypes in the analysis included 1) CD8 as a T cell corrector predominantly expressed on cytotoxic $\mathrm{T}$ cells; 2) CD45RO as a transmembrane glycoprotein expressed largely on previously activated or memory T cells; and 3) forkhead box P3 (FOXP3) as a transcriptional regulator which is crucial for the development and inhibitory function of natural $\mathrm{T}$ regulatory (Treg) cells and adaptive or induced Treg cells. This selection was based on the results of a previous systematic review that suggested that these subtypes were initially important in the prognosis of colorectal cancer (Pagès et al. 2005; Salama et al. 2009; Nosho et al. 2010; Ladoire et al. 2011; Mlecnik et al. 2011; Roxburgh and McMillan 2012; Väyrynen et al. 2013; Ohno et al. 2014). The CD66b+ specifically expressed in neutrophils is widely used to investigate neutrophil infiltration in different types of human cancer, such as renal cell carcinomas, hepatocellular carcinomas, non-small cell lung cancers, melanoma, and colorectal cancers (Rao et al. 2012).

Stored paraffin-embedded blocks of the CLM specimens were retrieved to perform immunohistochemistry. Representative areas of the most invasive tumor were selected from each. Consecutively, blank $4-\mu$ m-thick sections were cut and mounted on silanized slides that were dewaxed in xylene and rehydrated using graded alcohol washes. Heat-induced antigen retrieval was performed by the microwaving method under pressure using citrate or Tris/ethylene diamine tetraacetic acid (EDTA) buffer pretreated. Prior to microwaving, the endogenous peroxidase activity was blocked with 5\% hydrogen peroxide, and the following primary antibodies were applied: CD8 (clone: C8/144B, Dako), CD45RO (clone: UCHL1, Dako), FOXP3 
(clone: 236A/E7, eBioscience) and CD66b (clone: G10F5BD, Pharmingen). Sections were washed twice with TBS and incubated with Dako Envision, and 3,3'-diaminobenzidine (DAB) was eventually applied to indicate positive expression. Finally, sections were counterstained with hematoxylin, dehydrated, and mounted on slides.

The CLM tissues of the 29 patients were immunohistochemically analyzed for immune-cell infiltration at the invasive margin of the tumor. The number of CD8+, $\mathrm{CD} 45 \mathrm{RO}+, \mathrm{FOXP} 3+$, or $\mathrm{CD} 66 \mathrm{~b}+$ cells was randomly counted under $\times 200$ magnification in six parts of the marginal tumor, and the median number in each part was determined. Staining showing the number of cells to be over the median was defined as high numbers (Fig. 1 A-D), whereas that showing the number of cells to be lower than the median number was defined as low numbers of infiltrated cells. The positive control was defined as lymphocytes from a specimen of spleen provided by pathologist H.K. The inflammatory cells were counted by four independent observers including a professor of pathology at our institution (co-author H.K.), and each observer was blinded to the clinicopathological features of the patient associated with each specimen.

Correlation between peripheral blood neutrophils and TILs

The correlation between systemic immune responses and local immune responses was evaluated by using the pre-operative peripheral blood NLR and the number of TILs. The number of peripheral blood neutrophils was analyzed in relation to the number of the TILs, such as CD8+ T cells, CD45RO $+\mathrm{T}$ cells, and Foxp $3+\mathrm{T}$ cells, and CD66b + neutrophils.

\section{Statistical analysis}

Data are expressed as the mean \pm SD. Data from different groups were compared using one-way analysis of variance (ANOVA) and examined by Student $t$-test or Dunnett's multiple comparison test. Comparisons between groups were performed with Chi-square tests. KaplanMeier survival curves with log-rank tests were used to visualize differences of OS for patient groups. Only univariate analysis was performed for prognostic analysis. Rates of OS and cancer-specific survival were calculated. A twotailed $\mathrm{p}$ value $<0.05$ was considered significant. The Statistical Package for JMP ${ }^{\circledR} 11$ (SAS Institute Inc., Cary, $\mathrm{NC}, \mathrm{USA}$ ) was used for all statistical analyses.

\section{Results}

\section{Patient clinicopathological demographics}

In total, 29 patients who underwent hepatic resections for CLM in this study were analyzed. Table 1 summarizes the clinicopathological characteristics of the patients. Of them, 20 patients $(69 \%)$ were men and $9(31 \%)$ were women. The mean age of the patients at the time of surgery

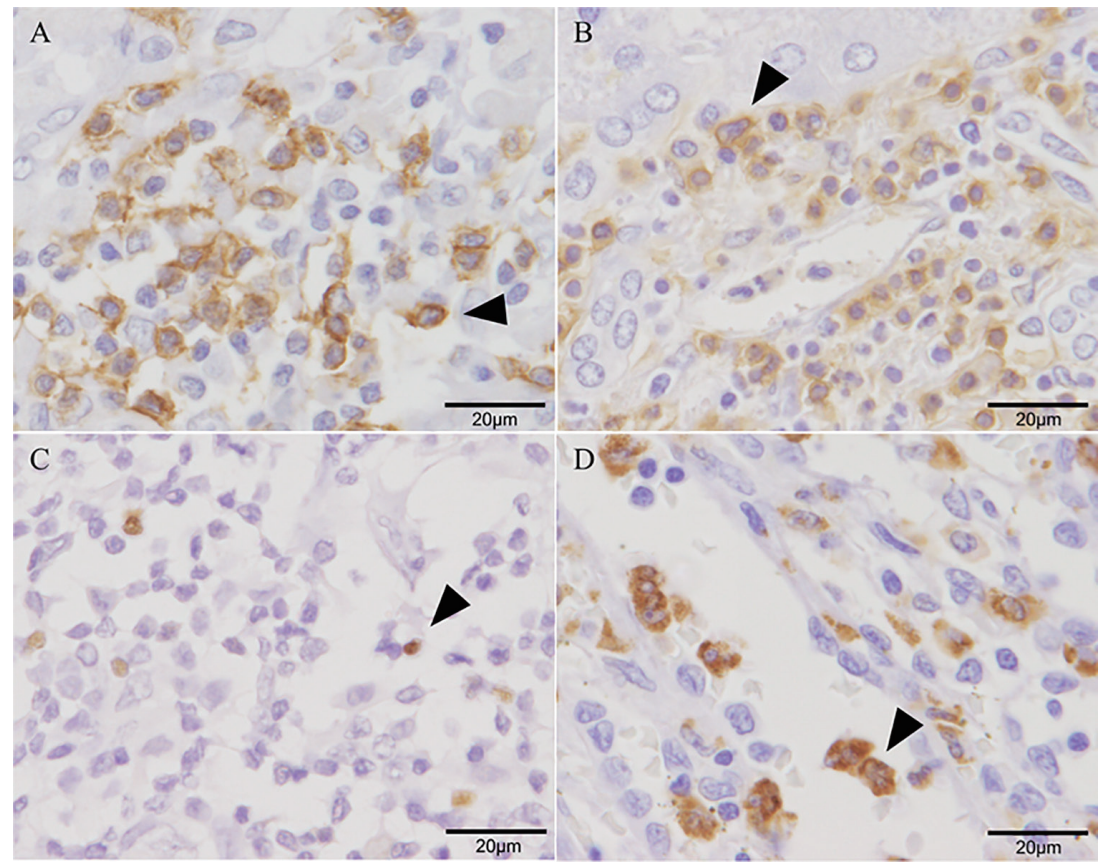

Fig. 1. Representative images of infiltrated TILs in CLM tissue specimens.

(A) Positive expression of CD8 (arrow) in the cell membrane of lymphocytes in a tissue specimen derived from a 58-year-old male patient with metachronous CLM representing high numbers $(\times 400)$, (B) Positive expression of $\mathrm{CD} 45 \mathrm{RO}$ (arrow) in the cell membrane of lymphocytes derived from a 79-year-old male patient with metachronous CLM representing high numbers $(\times 400)$, (C) Positive expression of FOXP3 (arrow) in the nuclei of lymphocytes derived from a 67-year-old female patient with metachronous CLM representing high numbers $(\times 400)$, and $(D)$ Positive expression of CD66b (arrow) in the cell membrane of neutrophils derived from a 47-year-old female patient with synchronous CLM representing high numbers $(\times 400)$. 
was $63 \pm 11.6$ years (range 41-83 years). Four patients $(13 \%)$ had synchronous metastases. The mean number of liver metastases was $2 \pm 3.0$ (range 1-14). The mean maximal size of the liver metastasis was $4.5 \pm 2.5 \mathrm{~cm}$ (range $1-12.5 \mathrm{~cm})$. Five patients $(18 \%)$ underwent a hemihepatectomy, 12 patients $(41 \%)$ underwent sectionectomy or segmentectomy, and 12 patients (41\%) underwent limited resections.

Ultimately, 20 patients $(65 \%)$ died from colorectal cancer, and 9 patients (35\%) were still alive as of 2018. Among these 9 patients, 2 had suffered the recurrence of intrahepatic metastasis. The median follow-up period for the survivors in this study was 51 months (range 2-97 months). The 1-, 3- and 5-year cancer-specific OS rates were $86 \%, 62 \%$ and $44 \%$, respectively.

\section{Comparison between survival and each parameter}

Table 2 shows the univariate analysis for cancer-specific survivals. The factors of the number of tumors less than four metastases, absence of extra-hepatic metastasis, adjuvant chemotherapy, and an elevated NLR over 4.1 (mean value) were significantly correlated with better cancer-specific survival $(\mathrm{p}<0.05)$. The systemic inflammatory biomarkers assessed by the mGPS were not significantly associated with cancer-specific survival in this study.

\section{Relationship between immune cell infiltration and prognosis}

Median cut-off values of positivity were set as follows: CD8 $+\mathrm{T}$ cells, 8.2; CD45RO $+\mathrm{T}$ cells, 40.3; FOXP3 $+\mathrm{T}$ cells, 14.7; and CD66b+ neutrophils, 25.7. The categorical data were also divided into low- and high-number groups. Survival analysis showed that CLM patients $(\mathrm{n}=13)$ with

Table 1. Patients demographics and clinicopathological features $(n=29)$.

\begin{tabular}{ll}
\hline Age, year (range) & $63(41-83)$ \\
Sex (Male : Female) & $20(69 \%): 9(31 \%)$ \\
Time of liver metastasis (Synchronous : Metachronous) & $4(14 \%): 25(86 \%)$ \\
Extrahepatic metastasis (no : yes) & $24(83 \%): 5(17 \%)$ \\
Number of liver metastases (range) & $2(1-14)$ \\
Maximal liver metastases (cm) (range) & $4.47(1-12.5)$ \\
Adjuvant chemotherapy (yes : no) & $15(52 \%): 14(48 \%)$ \\
Recurrence in residual liver (yes : no) & $9(52 \%): 20(48 \%)$ \\
\hline
\end{tabular}

Table 2. Relationship between overall survivals and clinico-pathological findings or immune-histochemical results.

\begin{tabular}{|c|c|c|c|}
\hline Variables & Number of patients & $\begin{array}{c}\text { Hazard ratio } \\
(95 \% \text { coefficient incidence })\end{array}$ & $\begin{array}{l}\text { Significance } \\
\text { (p value) }\end{array}$ \\
\hline \multicolumn{4}{|l|}{ Factors of primary site } \\
\hline \multicolumn{4}{|l|}{ Histological differentiation } \\
\hline well/moderately & $28 / 1$ & $0.97(0.19-17.68)$ & 0.977 \\
\hline \multicolumn{4}{|l|}{ Lymph node metastasis } \\
\hline positive/negative & $18 / 9$ & $0.94(0.32-2.49)$ & 0.917 \\
\hline \multicolumn{4}{|l|}{ Factors of liver metastases } \\
\hline \multicolumn{4}{|l|}{ Time of metastasis } \\
\hline synchronous/metachronous & $4 / 25$ & $1.51(0.35-4.65)$ & 0.530 \\
\hline \multicolumn{4}{|l|}{ Number of tumor } \\
\hline$<4 / \geqq 4$ & $22 / 7$ & $0.13(0.04-0.39)$ & 0.0005 \\
\hline \multicolumn{4}{|l|}{ Extrahepatic metastasis } \\
\hline absent/present & $24 / 5$ & $0.22(0.07-0.75)$ & 0.018 \\
\hline \multicolumn{4}{|l|}{ Adjuvant chemotherapy } \\
\hline yes/no & $14 / 15$ & $0.19(0.06-0.54)$ & 0.0013 \\
\hline \multicolumn{4}{|l|}{ Factors of host responses } \\
\hline Modified Glasgow Prognostic Score $0 / 1 / 2$ & $24 / 3 / 2$ & & \\
\hline 0 vs. 1 & $24 / 3$ & $0.51(0.16-2.21)$ & 0.326 \\
\hline 0 vs. 2 & $24 / 2$ & $0.90(0.17-15.81)$ & 0.896 \\
\hline \multicolumn{4}{|l|}{ Neutrophil to lymphocyte ratio (NLR) } \\
\hline$\geqq 4.1 /<4.1$ & $3 / 26$ & $0.17(0.05-0.78)$ & 0.026 \\
\hline
\end{tabular}


only high numbers of CD45RO+ memory $\mathrm{T}$ cells had significantly longer survival in comparison with those with low numbers $(n=16)(p=0.02$, Fig. 2$)$. The degree of infiltration of CD8 + T cells, FOXP3 + T cells, or CD66b+ neutrophils was not significantly associated with prognosis in patients died of colorectal cancer (Fig. 2).

\section{Correlation between peripheral blood neutrophils and TILs}

Fig. 3 shows the correlations between numbers of infiltrated CD45RO $+\mathrm{T}$ cells and other immune cells (CD8+ $\mathrm{T}$ cells, FOXP $3+\mathrm{T}$ cells and CD66b + neutrophils). Among them, the number of $\mathrm{CD} 45 \mathrm{RO}+\mathrm{T}$ cells was significantly correlated with that of CD8+ T cells $(p<0.05)$.

To evaluate the correlation between systemic and local immune responses, we examined the correlation between the numbers of peripheral blood neutrophils and the numbers of tumor-infiltrating lymphocytes $(\mathrm{CD} 8+\mathrm{T}$ cells, FOXP3 $+\mathrm{T}$ cells and CD45RO+ T cells) in metastatic liver cancer tissue (Fig. 4). The high numbers of neutrophils in the peripheral blood were significantly correlated with the high numbers of CD66b+ neutrophils in the CLM (Fig. 4). The number of neutrophils in the peripheral blood was inversely correlated with the number of $\mathrm{CD} 45 \mathrm{RO}+\mathrm{T}$ cells, and this was identified as a potentially prognostic parameter of longer survival than those described above. There was no correlation between neutrophils in peripheral blood and the number of infiltrated CD8+ T cells or FOXP3 $+\mathrm{T}$ cells in the metastatic tumor.

\section{Discussion}

Several studies have shown that the complexities of the tumor inflammatory microenvironment and host immune responses to co-existing tumors are important factors in patients with colorectal cancers (Pagès et al. 2005; Galon et al. 2006; Salama et al. 2009; Nosho et al. 2010; Ladoire et al. 2011; Mlecnik et al. 2011; Roxburgh and McMillan 2012; Väyrynen et al. 2013; Richards et al. 2014). However, liver metastases originating from colorectal carcinoma were considered to be a less immunogenic condition because cancer progression or metastasis may already activate the immune system in the primary regions (Wagner et al. 2008). The present study showed the importance of the host immune response in the regulation of tumor progression in CLM.

Our study showed that the NLR, but not the mGPS, has prognostic significance in patients who underwent hepatic resections for CLM. As in the recent study by McMillan et al. (2007), elevated inflammatory markers,
CD8+ cytotoxic T cell

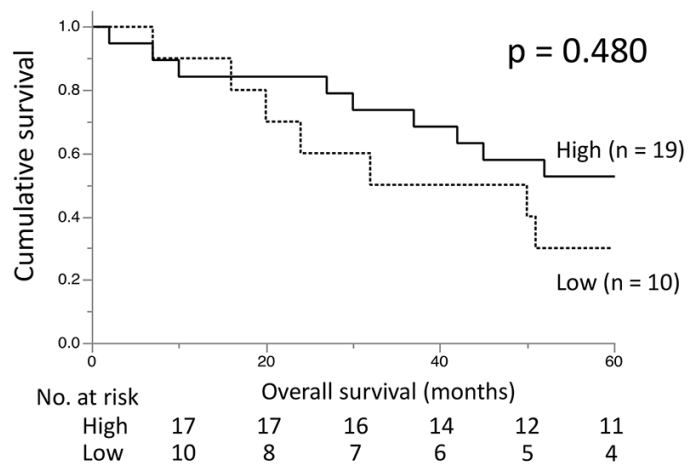

FOXP3+ regulatory T cell

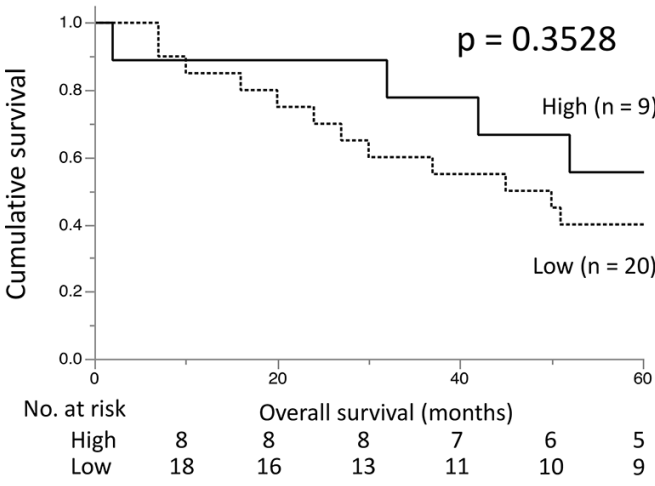

CD45RO+ memory T cell

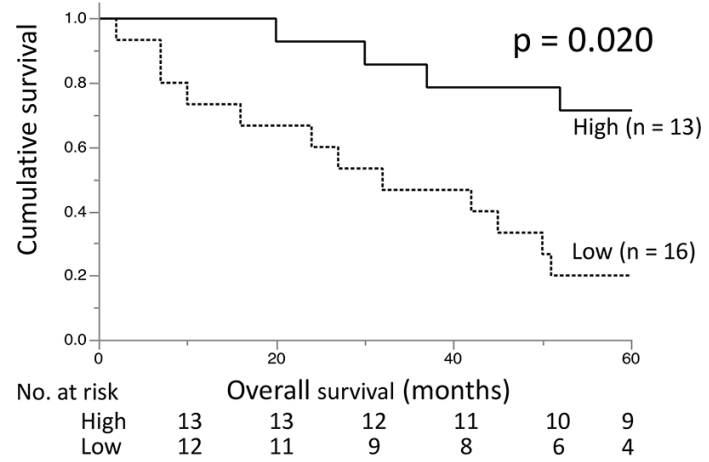

CD66b+ neutrophils

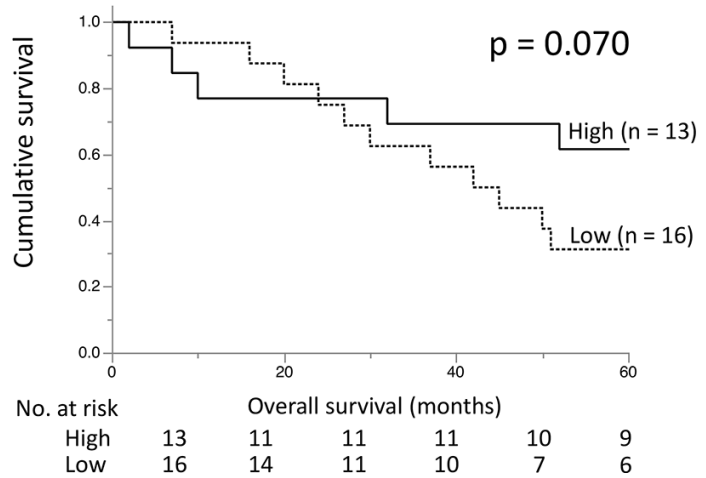

Fig. 2. Overall rates of survival of CLM patients.

Each graph shows overall rates of survival of CLM patients with high (solid line) or low (dotted line) numbers of CD8+ cytotoxic T cell, CD45RO+ memory T cell, FOXP3 + regulatory T cell and CD66b+ neutrophils after hepatectomy. The numbers, shown at bottom, indicate the numbers of survivors in each group of high or low numbers at every 10 months after hepatectomy. 
T. Hamada et al.
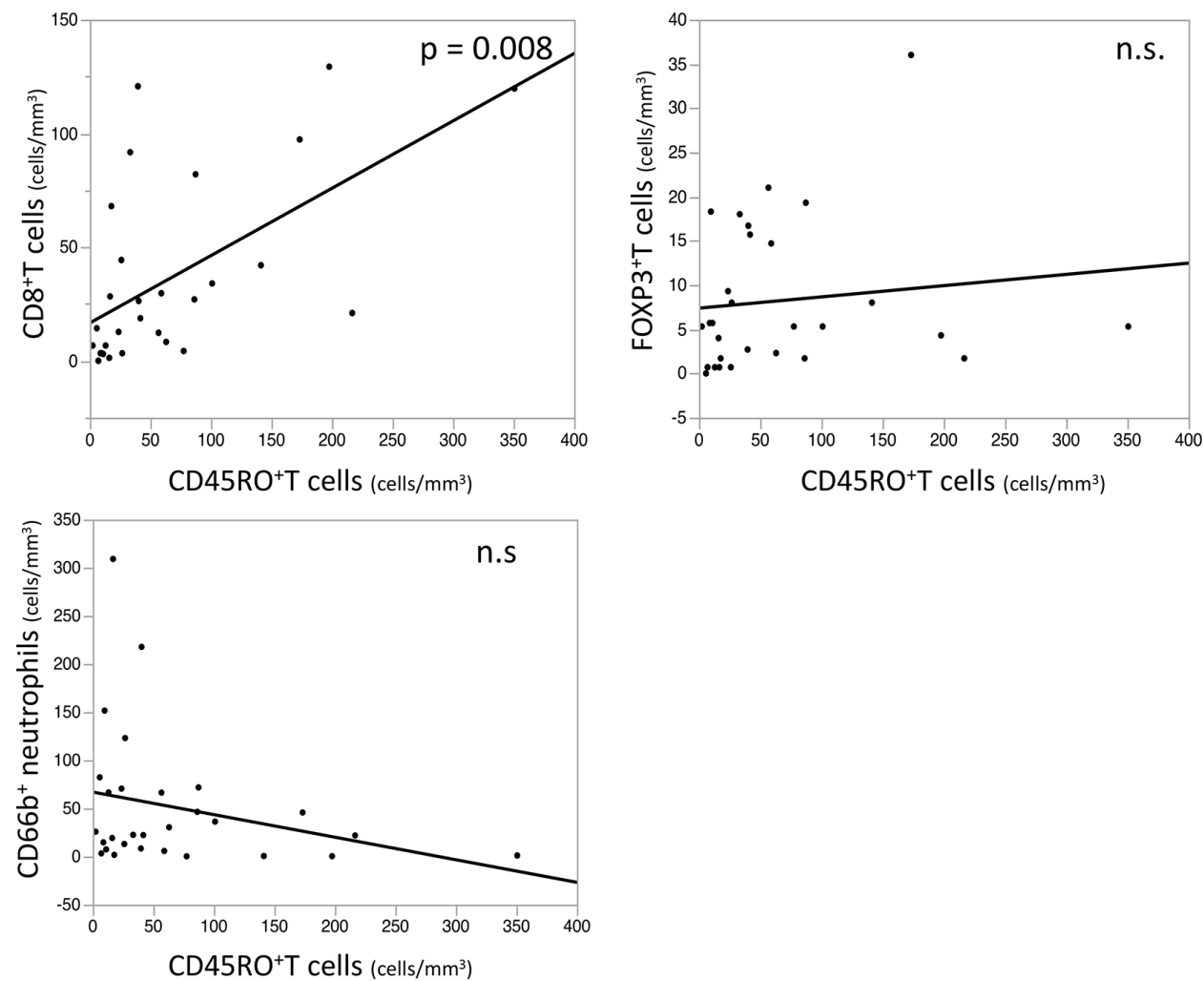

Fig. 3. Correlation of expression between CD45RO+ T cells and other TILs.

The number of patients for each graph was 29 .

n.s., not significant.
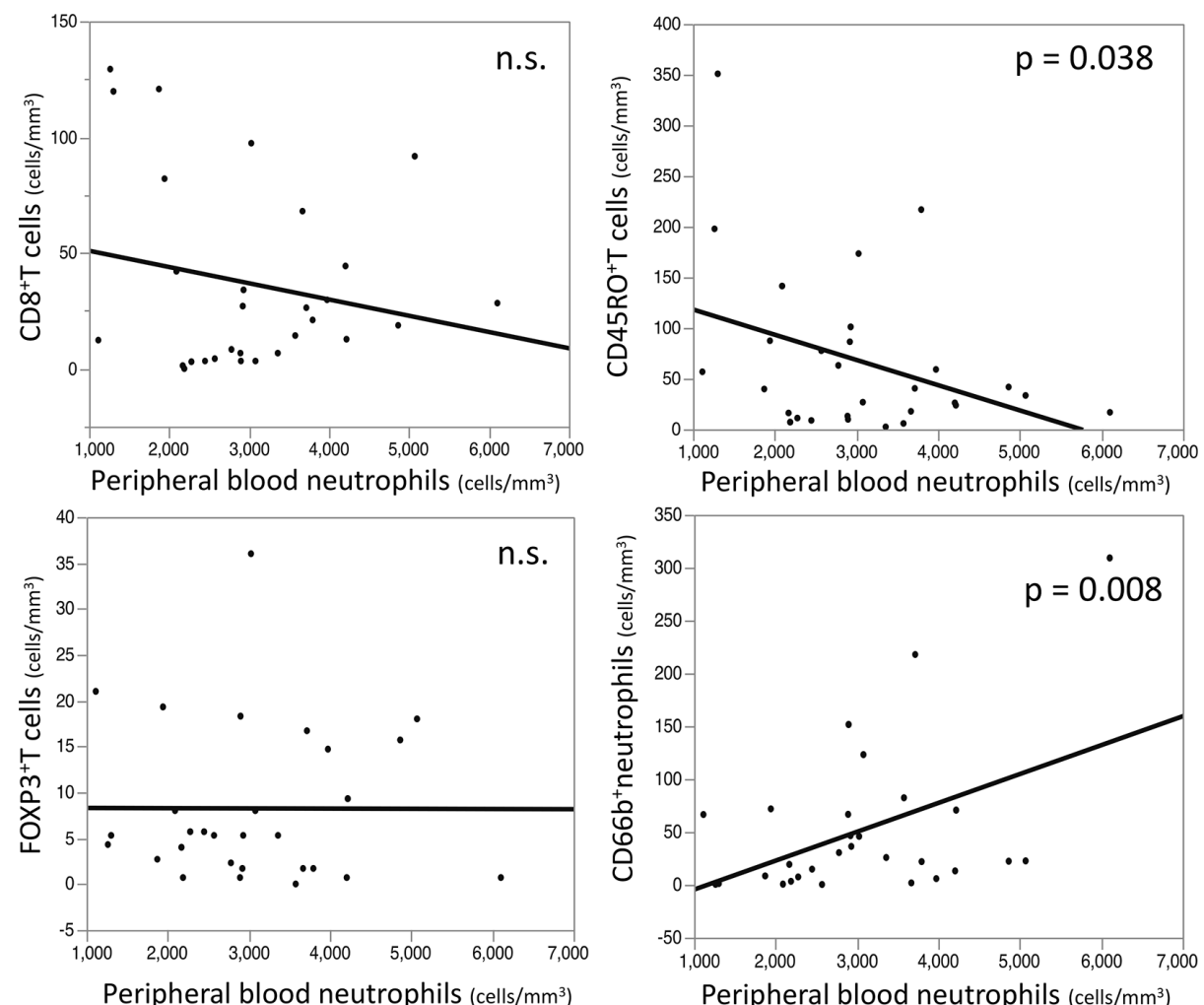

Fig. 4. Correlation of positivity between peripheral blood neutrophils and TILs. The number of patients for each graph was 29 . n.s., not significant. 
especially that of C-reactive protein, have been used as prognostic tools in patients undergoing curative resection for primary colorectal cancers. The additional preoperative marker of increased NLR was associated with poor prognosis in the patients in our study. Furthermore, some other studies showed a relationship between increased NLR and patient survival outcomes after resection of CLM (Halazun et al. 2008; Chang et al. 2014). In the present study, only the NLR as it relates to factors of host response showed prognostic significance in patients with resected CLM. This indicates that systemic immune response as indicated by the NLR may be more sensitive than other markers of this response. Neutrophils have recently emerged as important tumor-infiltrating myeloid cells, playing a role in tumor growth and progression (Galdiero et al. 2013). Neutrophils contain and secrete growth factors or cytokines such as VEGF, TGF- $\beta$, and pro-angiogenic factors, which are thought to play an integral role in tumor development (Mantovani et al. 2011). Increased neutrophil count may aid in the development and progression of neoplasms by providing an adequate environment for growth, and an elevated NLR may confer a survival advantage for CLM.

We first conducted this study to examine the independent prognostic value of tumor-infiltrating immune cell subsets in patients with CLM who underwent hepatectomy. In agreement with previous reports, the present study confirmed the significance of the CD45RO $+\mathrm{T}$ cells as a candidate useful as predictive factors of survival (Pagès et al. 2009; Nosho et al. 2010; Galon et al. 2012; Lee et al. 2013). The novel finding in the current study is the correlation of the high number of $\mathrm{CD} 45 \mathrm{RO}+\mathrm{T}$ cells at the metastatic colon cancer site with better prognosis. The prognostic impact of $\mathrm{CD} 45 \mathrm{RO}+\mathrm{T}$ cells at the site of the liver metastasis has not been investigated in detail so far. The present result regarding the significant association between increased numbers of CD45RO+ memory $\mathrm{T}$ cells in CLM and patient survival after hepatectomy was of great importance in this study. The mechanism of the anti-tumor reaction in TILs is still controversial. However, memory T cells can maintain anti-cancer reactions for a long period after primary stimulation in the primary cancer lesions, as reported previously, and may respond rapidly upon reexposure to antigens from a metastatic recurrence (Pagès et al. 2009; Lee at al. 2013; Hu and Wang 2017). The trafficking properties and the long-lasting anti-tumor capacity of memory $\mathrm{T}$ cells could result in long-term immunity in human cancer (Galon et al. 2012). Moreover, a recent study reported that infiltration of $\mathrm{CD} 45 \mathrm{RO}+\mathrm{T}$ cells was associated with microsatellite instability and DNA methylation, which may play roles in the prognosis of colorectal cancer patients (Ogino et al. 2008, Nosho et al. 2010). Although the precise mechanism of the anti-cancer effects of $\mathrm{CD} 45 \mathrm{RO}+$ memory $\mathrm{T}$ cells in various cancers remains unclear, possible explanations have been surmised as follows: it may partially relate to the features of $\mathrm{CD} 45 \mathrm{RO}+\mathrm{T}$ cells, as they (1) are the hallmark of adaptive immunity; (2) display a low-activation threshold; (3) vigorously proliferate despite minimal co-stimulation; and (4) persist over a life-time with stem cell-like multipotency and self-renewal characteristics (Hu and Wang 2017). To clarify the realworld value of this study, the association between NLR or memory $\mathrm{T}$ cells and patient survival needs to be investigated in a larger sample size.

Our study also showed that elevated neutrophils in peripheral blood were significantly correlated with lower numbers of CD45RO+ $\mathrm{T}$ cells in metastatic liver tumors. This may suggest that both systemic and local immune responses are in a causal and close relationship. Okano et al. (2003) found that CLM patients with less dense lymphocytic infiltration at the tumor margin had a poorer prognosis after liver resection than those with a greater density of infiltration. Although the results in the present study were limited by its retrospective nature and the small sample size, further investigation may show the precise mechanisms of the anti-tumor response and clinical manifestations with respect to patient prognosis.

In conclusion, the present study showed that the host's immune responses as represented by elevated NLR in peripheral blood and high numbers of intratumoral $\mathrm{CD} 45 \mathrm{RO}+\mathrm{T}$ cell may have significant role in inhibiting tumor progression. This is the first study, to the best of our knowledge, to address the relationship between elevated NLR in peripheral blood or increased numbers of $\mathrm{CD} 45 \mathrm{RO}+\mathrm{T}$ cells in metastatic cancer tissues and survival in patients who underwent hepatectomy for CLM. These immunological markers could potentially be applied in the future to identify high-risk patients and select adjuvant chemo- or immunotherapy for preventing early cancer relapse.

\section{Acknowledgments}

This article is dedicated to the memory of former Professor Kazuo Chijiiwa, who initially directed the present study but passed away on November 26, 2013, before the entire study could be completed.

This work was supported by institutional grants from the University of Miyazaki Faculty of Medicine (Approval number \#2014-028).

\section{Conflict of Interest}

The authors declare no conflict of interest.

\section{References}

Beppu, T., Sakamoto, Y., Hasegawa, K., Honda, G., Tanaka, K., Kotera, Y., Nitta, H., Yoshidome, H., Hatano, E., Ueno, M., Takamura, H., Baba, H., Kosuge, T., Kokudo, N., Takahashi, K., et al. (2012) A nomogram predicting disease-free survival in patients with colorectal liver metastases treated with hepatic resection: multicenter data collection as a Project Study for Hepatic Surgery of the Japanese Society of Hepato-BiliaryPancreatic Surgery. J. Hepatobiliary Pancreat. Sci., 19, 72-84.

Bindea, G., Mlecnik, B., Fridman, W.H., Pagès, F. \& Galon, J. (2010) Natural immunity to cancer in humans. Curr. Opin. 
Immunol., 22, 215-222.

Chang, Z., Zheng, J., Ma, Y., Zhao, J., Wang, C. \& Liu, Z. (2014) The neutrophil-to-lymphocyte ratio as a predictor for recurrence of colorectal liver metastases following radiofrequency ablation. Med. Oncol., 31, 855.

Galdiero, M.R., Bonavita, E., Barajon, I., Garlanda, C., Mantovani, A. \& Jaillon, S. (2013) Tumor associated macrophages and neutrophils in cancer. Immunobiology, 218, 1402-1410.

Galon, J., Costes, A., Sanchez-Cabo, F., Kirilovsky, A., Mlecnik, B., Lagorce- Pagès, C., Tosolini, M., Camus, M., Berger, A., Wind, P., Zinzindohoué, F., Bruneval, P., Cugnenc, P.H., Trajanoski, Z., Fridman, W.H., et al. (2006) Type, density, and location of immune cells within human colorectal tumors predict clinical outcome. Science, 313, 1960-1964.

Galon, J., Pagès, F., Marincola, F.M., Thurin, M., Trinchieri, G., Fox, B.A., Gajewski, T.F. \& Ascierto, P.A. (2012) The immune score as a new possible approach for the classification of cancer. J. Transl. Med., 10, 1.

Halazun, K.J., Aldoori, A., Malik, H.Z., Al-Mukhtar, A., Prasad, K.R., Toogood, G.J. \& Lodge, J.P. (2008) Elevated preoperative neutrophil to lymphocyte ratio predicts survival following hepatic resection for colorectal liver metastases. Eur. J. Surg. Oncol., 34, 55-60.

Hu, G. \& Wang, S. (2017) Tumor-infiltrating CD45RO(+) memory $\mathrm{T}$ lymphocytes predict favorable clinical outcome in solid tumors. Sci. Rep., 7, 10376.

Jemal, A., Siegel, R., Xu, J. \& Ward, E. (2010) Cancer statistics, 2010. CA Cancer J. Clin., 60, 277-300.

Kimura, Y., Morohashi, S., Yoshizawa, T., Suzuki, T., Morohashi, H., Sakamoto, Y., Koyama, M., Murata, A., Kijima, H. \& Hakamada, K. (2016) Clinicopathological significance of vascular endothelial growth factor, thymidine phosphorylase and microvessel density in colorectal cancer. Mol. Med. Rep., 13, 1551-1557.

Klintrup, K., Makinen, J.M., Kauppila, S., Vare, P.O., Melkko, J., Tuominen, H., Tuppurainen, K., Makela, J., Karttunen, T.J. \& Makinen, M.J. (2005) Inflammation and prognosis in colorectal cancer. Eur. J. Cancer, 41, 2645-2654.

Ladoire, S., Martin, F. \& Ghiringhelli, F. (2011) Prognostic role of FOXP3+ regulatory T cells infiltrating human carcinomas: the paradox of colorectal cancer. Cancer Immunol. Immunother., 60, 909-918.

Lee, W.S., Kang, M., Baek, J.H., Lee, J.I. \& Ha, S.Y. (2013) Clinical impact of tumor-infiltrating lymphocytes for survival in curatively resected stage IV colon cancer with isolated liver or lung metastasis. Ann. Surg. Oncol., 20, 697-702.

Lunevicius, R., Nakanishi, H., Ito, S., Kozaki, K., Kato, T., Tatematsu, M. \& Yasui, K. (2001) Clinicopathological significance of fibrotic capsule formation around liver metastasis from colorectal cancer. J. Cancer Res. Clin. Oncol., 127, 193-199.

Malik, H.Z., Prasad, K.R., Halazun, K.J., Aldoori, A., Al-Mukhtar, A., Gomez, D., Lodge, J.P. \& Toogood, G.J. (2007) Preoperative prognostic score for predicting survival after hepatic resection for colorectal liver metastases. Ann. Surg., 246, 806-814.

Mantovani, A., Cassatella, M.A., Costantini, C. \& Jaillon, S. (2011) Neutrophils in the activation and regulation of innate and adaptive immunity. Nat. Rev. Immunol., 11, 519-531.

McMillan, D.C., Crozier, J.E., Canna, K., Angerson, W.J. \& McArdle, C.S. (2007) Evaluation of an inflammation-based prognostic score (GPS) in patients undergoing resection for colon and rectal cancer. Int. J. Colorectal Dis., 22, 881-886.

Mlecnik, B., Tosolini, M., Kirilovsky, A., Berger, A., Bindea, G., Meatchi, T., Bruneval, P., Trajanoski, Z., Fridman, W.H., Pagès, F. \& Galon, J. (2011) Histopathologic-based prognostic factors of colorectal cancers are associated with the state of the local immune reaction. J. Clin. Oncol., 29, 610-618.
Nosho, K., Baba, Y., Tanaka, N., Shima, K., Hayashi, M., Meyerhardt, J.A., Giovannucci, E., Dranoff, G., Fuchs, C.S. \& Ogino, S. (2010) Tumour-infiltrating T-cell subsets, molecular changes in colorectal cancer, and prognosis: cohort study and literature review. J. Pathol., 222, 350-366.

Ogino, S., Nosho, K., Kirkner, G.J., Kawasaki, T., Chan, A.T., Schernhammer, E.S., Giovannucci, E.L. \& Fuchs, C.S. (2008) A cohort study of tumoral LINE-1 hypomethylation and prognosis in colon cancer. J. Natl. Cancer Inst., 100, 1734-1738.

Ohno, A., Yorita, K., Haruyama, Y., Kondo, K., Kato, A., Ohtomo, T., Kawaguchi, M., Marutuska, K., Chijiiwa, K. \& Kataoka, H. (2014) Aberrant expression of monocarboxylate transporter 4 in tumour cells predicts an unfavourable outcome in patients with hepatocellular carcinoma. Liver Int., 34, 942-952.

Okano, K., Maeba, T., Moroguchi, A., Ishimura, K., Karasawa, Y., Izuishi, K., Goda, F., Usuki, H., Wakabayashi, H. \& Maeta, H. (2003) Lymphocytic infiltration surrounding liver metastases from colorectal cancer. J. Surg. Oncol., 82, 28-33.

Pagès, F., Berger, A., Camus, M., Sanchez-Cabo, F., Costes, A., Molidor, R., Mlecnik, B., Kirilovsky, A., Nilsson, M., Damotte, D., Meatchi, T., Bruneval, P., Cugnenc, P.H., Trajanoski, Z., Fridman, W.H., et al. (2005) Effector memory $\mathrm{T}$ cells, early metastasis, and survival in colorectal cancer. $N$. Engl. J. Med., 353, 2654-2666.

Pagès, F., Kirilovsky, A., Mlecnik, B., Asslaber, M., Tosolini, M., Bindea, G., Lagorce, C., Wind, P., Marliot, F., Bruneval, P., Zatloukal, K., Trajanoski, Z., Berger, A., Fridman, W.H. \& Galon, J. (2009) In situ cytotoxic and memory T cells predict outcome in patients with early-stage colorectal cancer. J. Clin. Oncol., 27, 5944-5951.

Popat, S., Hubner, R. \& Houlston, R.S. (2005) Systematic review of microsatellite instability and colorectal cancer prognosis. $J$. Clin. Oncol., 23, 609-618.

Rao, H.L., Chen, J.W., Li, M., Xiao, Y.B., Fu, J., Zeng, Y.X., Cai, M.Y. \& Xie, D. (2012) Increased intratumoral neutrophil in colorectal carcinomas correlates closely with malignant phenotype and predicts patients' adverse prognosis. PLoS One, 7, e30806.

Richards, C.H., Roxburgh, C.S., Powell, A.G., Foulis, A.K., Horgan, P.G. \& McMillan, D.C. (2014) The clinical utility of the local inflammatory response in colorectal cancer. Eur. J. Cancer, 50, 309-319.

Roxburgh, C.S. \& McMillan, D.C. (2012) The role of the in situ local inflammatory response in predicting recurrence and survival in patients with primary operable colorectal cancer. Cancer Treat. Rev., 38, 451-466.

Roxburgh, C.S., Salmond, J.M., Horgan, P.G., Oien, K.A. \& McMillan, D.C. (2009) Comparison of the prognostic value of inflammation-based pathologic and biochemical criteria in patients undergoing potentially curative resection for colorectal cancer. Ann. Surg., 249, 788-793.

Salama, P., Phillips, M., Grieu, F., Morris, M., Zeps, N., Joseph, D., Platell, C. \& Iacopetta, B. (2009) Tumor-infiltrating FOXP3+ $\mathrm{T}$ regulatory cells show strong prognostic significance in colorectal cancer. J. Clin. Oncol., 27, 186-192.

Salazar, R., Roepman, P., Capella, G., Moreno, V., Simon, I., Dreezen, C., Lopez-Doriga, A., Santos, C., Marijnen, C., Westerga, J., Bruin, S., Kerr, D., Kuppen, P., van de Velde, C., Morreau, H., et al. (2011) Gene expression signature to improve prognosis prediction of stage II and III colorectal cancer. J. Clin. Oncol., 29, 17-24.

Stoll, G., Bindea, G., Mlecnik, B., Galon, J., Zitvogel, L. \& Kroemer, G. (2015) Meta-analysis of organ-specific differences in the structure of the immune infiltrate in major malignancies. Oncotarget, 6, 11894-11909.

Tomlinson, J.S., Jarnagin, W.R., DeMatteo, R.P., Fong, Y., Kornprat, P., Gonen, M., Kemeny, N., Brennan, M.F., Blumgart, L.H. \& D'Angelica, M. (2007) Actual 10-year survival after resection of colorectal liver metastases defines cure. J. Clin. 
Oncol., 25, 4575-4580.

Väyrynen, J.P., Tuomisto, A., Klintrup, K., Makela, J., Karttunen, T.J. \& Makinen, M.J. (2013) Detailed analysis of inflammatory cell infiltration in colorectal cancer. Br. J. Cancer, 109, 1839-1847.

Wagner, P., Koch, M., Nummer, D., Palm, S., Galindo, L., Auten- rieth, D., Rahbari, N., Schmitz-Winnenthal, F.H., Schirrmacher, V., Buchler, M.W., Beckhove, P. \& Weitz, J. (2008) Detection and functional analysis of tumor infiltrating T-lymphocytes (TIL) in liver metastases from colorectal cancer. Ann. Surg. Oncol., 15, 2310-2317. 